\title{
Machine Learning Paradigm towards Content Based Image Retrieval on High Resolution Satellite Images
}

\author{
P.K.Kavitha, P.Vidhya Saraswathi
}

\begin{abstract}
In the current era, content based image retrieval based on pattern recognition and classification using machine learning paradigm is an innovative way. In order to retrieve high resolution satellite images Support Vector Machine (SVM) a machine learning paradigm is helpful for learning process and for pattern recognition and classification; ensemble methods give better machine learning results. In this paper, SVM based on random subspace and boosting ensemble learning is proposed for very high resolution satellite image retrieval. The learned SVM ensemble model is used to identify the images that most similar informative for active learning. A bias-weighting system is developed to direct the ensemble model to pay more attention on the positive examples than the negative ones. The UCMerced land use satellite image dataset is used for experimental work. Accuracy and error rate are found to be precise. The tentative effects illustrate that the proposed model derived enhanced retrieval accurateness at the optimum level as well as significantly more effective than existing approaches. The proposed method can diminish the gap dimensionality and conquer the difficulty. The comparisons are evaluated by using precision and recall measurements. Comparative analysis observed that the retrieval time for a particular image have been reduced and the precision is increased. The primary aim of this paper is to represent the significance of ensemble learning with support vector machine in efficient retrieval of image.
\end{abstract}

Keywords : Boosting, Ensemble learning, Machine learning, Random subspace, Support Vector Machine

\section{INTRODUCTION}

In Digital Image Processing content based image retrieval (CBIR) is a rising development for searching and retrieving the query image from large collection of databases. Two essential functions in a typical act of CBIR system are feature extraction and similarity measurement. The entire features of images are derived from all images in a database and it illustrates the substance of an image. To analyze the resemblance among the query image and the images in the database, similarity measurement function is used.

In the gadget of CBIR the principle venture is to symbolize every image in meticulous way to gives exact recognition of the image. The user enters the query image and all the

Revised Manuscript Received on December 15, 2019.

* Correspondence Author

P.K.Kavitha*, Department of Computer Applications, Kalasalingam Academy of Research and Education, Krishnankoil, Tamil Nadu, India. Email: kavitha.k@klu.ac.in

P. Vidhya Saraswathi, Department of Computer Applications, Kalasalingam Academy of Research and Education, Krishnankoil, Tamil Nadu, India. Email: vidhyasaraswathi.p@gmail.com features of query image are compared with the previously extracted and stored feature vectors of the database images. The similarity is made by evaluating the gap values and those vector values are used to rank the images as output in terms of their resemblance with the query images. At last, the majority similarly matching images are displayed as relevant output images. So, the success retrieval gadget depends on deciding on the proper image descriptors so as to pick the image accurately and length of feature vector. In this implement the preliminary classifier is used to recognize the majesty tag of the query image. After that scale the gap among the query image and the images in the database to retrieve most similar images.

Recently, Machine learning models are more accurate than typical models. The classification accuracy of image retrieval can be enhanced by ensemble method, which is a machine learning paradigm. So, SVM based ensemble learning is proposed in this paper.

The main contributions of our work is to proposed an effective machine learning framework for fast image retrieval that can be enhanced by ensemble learning method using SVM classifier. Compared to other learning methods SVM based ensemble learning perform base learning model and random subspace that generates diversity range throughout the feature selection. To mix a set of weak classifiers, boosting is used to change the training data weights. Our proposed model reduces the computational cost of retrieval significantly at the state-of-the art competence level.

The organization of this paper is as follows: Section II explains the related works. Section III gives an overview of methods and materials used for this work. In section IV, this article describes the proposed SVM based ensemble learning methodology with dataset description are reminded. Section $\mathrm{V}$ discusses the experimental results. Section VI provides conclusion of this paper.

\section{RELATED WORK}

Recent researches reported that classification accuracy can be enhanced through ensemble method. This method combines a set of weak learner models into a strong model [8][9]. In machine learning paradigm, most popular ensemble strategies are Bagging, random subspace and boosting. Boosting may create ensembles that are not as much of accurate than a single learner and bagging is approximately accurate than a single learner, that is sometimes much less accurate than boosting [7]. 
AdaBoost is the essentially used boosting method. Li, Wang, Sung offered AdaBoost SVM to resolve the classification problems [18]. Using AdaBoost approach is better while compared with the Decision Trees and Neural Networks classifiers. Zhou, et al. examined the performance of least squares SVM ensemble models for credit scoring [13].

These study shows that ensemble models can provide excellent solutions for many machine learning applications. Wang and Ma proposed RSB-SVM, that is based on SVM as a base learner and bagging and random subspace approaches also [7]. This technique exploit linear and polynomial kernel function. Aun Irtaza et al.[9] offered a genetic algorithm (GA) primarily based classifier comity learning (GCCL) scheme to discover fixed classifiers because of combining ANN with SVMs by asymmetric and symmetric bagging algorithm.

Haifeng Wang et al.[10] developed SVM-based ensemble learning algorithm on breast cancer analysis that uses to diminish the analysis variance and raise the accuracy of analysis. Different SVM models are mingled for analyze some dataset images.

Pouria Sadeghi et al.[20] offers an exceptionally scalable and computationally efficient image retrieval system for real-time content-based searching through large-scale image repositories within the domain of remote sensing and plant biology. Convolutional Neural Network is used as a function extractor to derive deep characteristic representations from the imaging data.

Lu Xu et al. [21] presented a stratified object-primarily based farmland extraction method. It includes two key procedures: one is image region division on a scale and the alternative is scale parameter pre-estimation inside the local regions. First, the entire region can be separated into different kind of regions primarily based on the texture functions, along with the homogeneity and the mean value.

Second, within neighborhood areas, the optimum spatial scale segmentation parameter changed into pre-envisioned by means of common neighborhood variance and its first-order and 2d-order rate of change. Through stratified regionalization and nearby segmentation parameters estimation, farmland segmentation may be accomplished. Images taken from GF-2 and Quickbird and mean-shift and multi-resolution segmentation algorithms were applied to prove the strength of the proposed technique.

Our proposed work is based on SVM based ensemble learning. In order to retrieve high resolution satellite images Support Vector Machine (SVM) a machine learning paradigm is helpful for learning process and for pattern recognition and classification, ensemble methods give better machine learning results.

In this paper, SVM based on random subspace and boosting ensemble learning is proposed for very high resolution satellite image retrieval. The learned SVM ensemble model is used to identify the images that most similar informative for active learning. A bias-weighting system is developed to direct the ensemble model to pay more attention on the positive examples than the negative ones.

\section{MATERIALS AND METHODS}

\section{A. Bagging}

This method was introduced by Breiman [5]. Bagging method creates different training data with replacing original data. Each training set, different classifiers are trained. Finally, based on majority voting, classifiers are combined into a absolute model. It can be able to produce enough training units, mainly supposed for facts deficiency. This is used to moderate the base classifiers variance and can avoid over fitting.

\section{B. Random Subspace}

Ensemble method based approach random subspace is first projected by Ho [7]. It is just like bagging in the sagacity that we bootstrap samples; conversely, the development is in use of feature space to construct a innovative training set. Repeating these steps numerous times, we can create many contradictory classifiers. Through easy majority voting, we obtain an ultimate representation.

\section{Boosting}

The explanation of this approach is to merge a set of weak classifiers into powerful classifiers. Classifier is weak or strong depends on the accuracy of the particular method. If the model is little better than guessing randomly, its called a weak classifier. A sequence of weak classifiers is identified by modifying the weight of instances that wrongly classified in the present iteration and have privileged weights in subsequently iteration; if not the inquiry weights are diminished. All weak classifiers classify the image data points, at last the predictions are completed. In the final model, more accurate classifiers will obtain high weights.

\section{Support Vector Machine}

In machine learning, support vector machines (SVM) are supervised learning models. Fig. 1 describes the algorithm of SVM classifier.

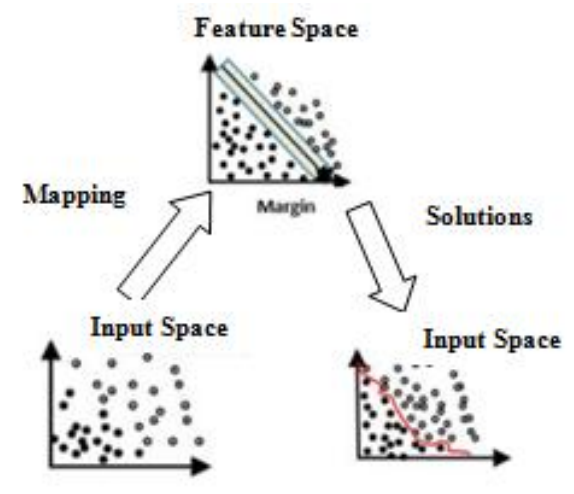

Fig. 1.SVM Algorithm

A set of data is given. Each data is manifest as any one contained in one of two classifications. SVM training algorithm creates a model that classifies a new data in one of these two classes. Basically, SVMs are designed for such binary classification; they are extendable for multi-class problems. 
The endeavor of SVM classification is to discover the optimal hyper plane that is used to differentiate group of vectors in a technique that one group of the necessary variables is on one side of hyper plane and the other class of variables are on the other side of plane. More rapidly close vectors to the hyper plane are called as support vectors. The most favorable hyper plane can be described as the linear classifier among the maximum margin for a given set of variables.

\section{E. Ensemble Learning}

A lot of ensemble classifiers are decayed into double forms of mechanism. In the first step, generate base classifiers with essential accuracy and diversity. The data splitting mechanism is used to complete this goal. Training algorithm of this method apply the equal range of parameter values on the dissimilar subsets of the training dataset.

For this splitting the data, we need partitioning method. Randomly divide the training dataset into equal-sized non-overlapping sets is known as partitioning data or splitting the data. Every data in the dataset has taken as a subset for training a base classifier.

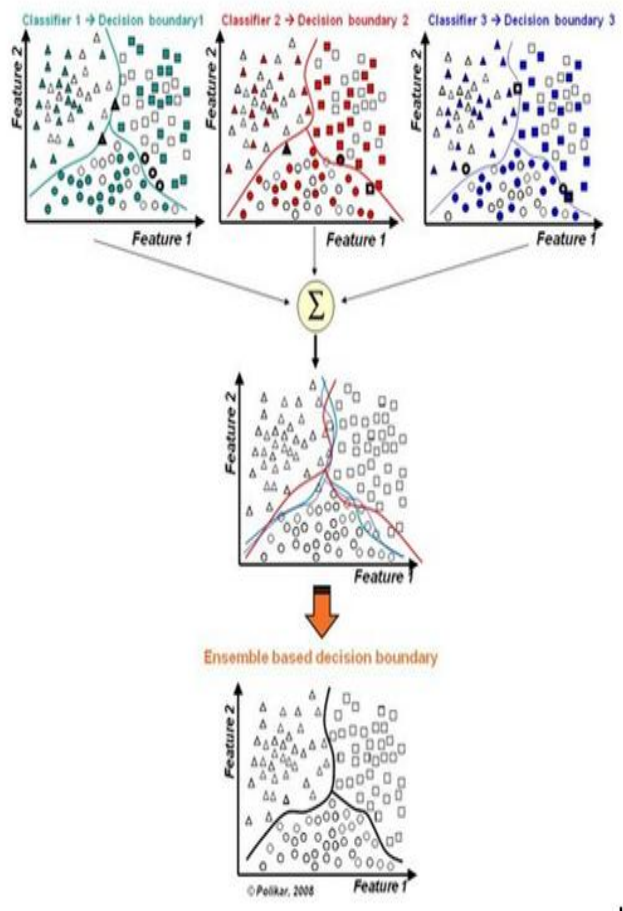

Fig. 2.Ensemble Learning

In the next module of ensemble combined every sub model outputs and calculate the weights as a numeric value. For compute the weighted average of base classifiers, bagging method is used. Then the weights are calculated and identical. This type of methods avoids several learning measures. Individual outputs of base classifiers introduce the more complex type of optimal mappings for learning base classifier models.

Also, ensemble method choose the optimal subsets of the base classifiers for best performance results[5]. A lot of methods exist for optimal subset selection. Only choose the best base classifiers for simplicity as the base model estimated by cross_validation.

\section{METHODOLOGY}

The proposed work has 2 phases namely training and testing. Most of the researches prove that ensemble approach can improve the classification accuracy.

Algorithm 1: Partitioning image data
Input: Training Image data
Output: Ensemble Model
For $\mathrm{n}=1$ to $\mathrm{N}$
Pick $\mathrm{p}(\mathrm{p}<\mathrm{r}) \%$ construct a new subset
$x_{\text {new }}=x_{11_{n}} x_{i 2} x_{i a_{n}} \ldots \ldots x_{l p}$
Choose the exact base algorithm $E_{\mathrm{n}}$ to the new subset
End
Output: New Ensemble model $\mathrm{E}=\operatorname{sign} \sum_{\mathrm{n}=1}^{N} E_{\mathrm{n}}(\mathrm{x})$

We proposed a novel machine learning paradigm using ensemble learning and SVM classifier for very high resolution satellite image retrieval. It uses SVM as a base classifier and choosing two popular ensemble approaches known as random subspace and boosting.

First one is random subspace that is a feature partitioning method and second one is boosting which is an instance partitioning method.

$$
\begin{aligned}
& \text { Algorithm 2: Combine Weak classifiers } \\
& \text { Input: Training Image data } \\
& T_{b}(\mathrm{x}) \text { is pick as the base classifier, } \\
& \quad \mathrm{B}=1,2,3 \ldots \ldots \mathrm{B} \\
& \text { First load the weight allotment of training data } \\
& D_{\mathrm{i}}=\frac{1}{\mathrm{~m}}, \quad \mathrm{i}=1,2,3 \ldots \mathrm{m} \\
& \quad \text { For b from } 1 \text { to B } \\
& \quad \text { Calculate the misclassification error rate } \\
& \varepsilon_{b}=P\left(T_{b}\left(x_{i}\right) \neq y_{i}\right) \\
& \quad \text { Compute coefficients of } T_{b}(\mathrm{x}) \\
& \alpha_{b}=\frac{1}{2} \log \frac{1-\varepsilon_{m}}{\varepsilon_{m}} \\
& \quad \text { Normalized constant } \\
& Z_{b}=\sum_{i=1}^{B} D_{i} \exp \left(-\alpha_{b} y_{i} T_{b}\left(x_{i}\right)\right) \\
& \quad \text { Update the weight } \\
& D_{i}=\frac{D_{i}}{z_{i}} \exp \left(-\alpha_{b} y_{i} T_{b}\left(x_{i}\right)\right) \\
& \text { End } \\
& \text { Output } \mathrm{T}(\mathrm{x})=\operatorname{sign}\left(\sum_{b=1}^{B} \alpha_{b} T_{b}(\mathrm{x})\right)
\end{aligned}
$$

In the first step, random subspace partitions the training sets into subsets by resembling feature, at that time, SVM was trained in sub data. Next, boosting boost up the instances of the given image. We decide a randomly selected subspace and then boosting the subspace as ensemble methods.

Published By: 
To partition original data for the training image, randomly selected subspace is used for creating samples. The image I has $\mathrm{n}$ occurrences.

$$
x_{l}=x_{1}, x_{2}, x_{3}, \ldots \ldots x_{n}
$$

Every instance has $f$ features. Each instance are represented by $l \& m$ features.

$$
x_{l m}=x_{l 1}, x_{l 2}, x_{l 3}, \ldots . . x_{l f}
$$

From the dataset image, we extract $p(p<r)$

$$
x_{\text {new }}=x_{l 1}, x_{l 2}, x_{l 3}, \ldots . . x_{l p}
$$

Random subspace gives special knowledge through selecting input data options. On the other hand, a series of classifiers combined for boosting that generates diversity. Typically, ensemble learning methods gives higher performance than a single model, totally different ensemble ways have different performance trusted on data sets.

The values from 0.01 to 100 are the range of parameter value. Appropriate base model with subsets, we obtain the range of SVM models in terms of Area under Curve (AUC). After that, we choose m models which have huge value of AUC.

\section{ALGORITHM 3: Ensemble Model \\ Input : Training image data \\ Output: Set of SVM Models \\ For parameters $\mathrm{N}=\mathrm{i}$ to $\mathrm{j} \quad \% \mathrm{i} \& \mathrm{j}$ are stable Choose the exact base algorithm $E_{n}$ to the data Compute equivalent AUC \\ End \\ Organize base algorithm Ap with respect to AUC Pick ' $n$ ' models by means of huge AUC \\ Resultant output: 'n' models $A_{n}$}

For classification problem, Boosting method is used. It frequently synthesizes weak classifiers. In addition, this approach has positive persuade in sturdy classifiers. By using the number of misclassifications dividing précis of instances, error rate is computed. Correlation between specificity and sensitivity explained by Receiver operating characteristic (ROC). In AUC, sensitivity illustrates the vertical axis and specificity denoted as horizontal axis. Area under the ROC curve contains the area curve with respect to horizontal axis. It ranges are within 0.5 to 1 . A superior value represents the higher model performance. It is a conventional method to assess the replica.

SVM algorithm cotains the following phases:

$$
\text { - Training }
$$

\section{Training Phase:}

Every input learning vector is represented by vectors. Input learning data for a SVM classifier consist of distance to border vectors, binary images. Vector $V_{i}$ with label $L_{i}$. $L_{i}= \pm 1,1 \leq i \leq S$, where $S$ is the number of samples. Fig.3 illustrates the training phase of proposed scheme.The decision boundary classify all points appropriately.

$$
L_{i}\left(E^{T} V_{i}+B\right) \geq 1, \forall i
$$

The assessment edge can be originate through solving the subsequent optimization problem:

$$
\text { Minimize } \frac{1}{2}\|E\|^{2} \text { subject to } L_{i}\left(E^{T} V_{i}+B\right) \geq 1, \forall i
$$

Lagrangian of this optimization problem is

$$
\operatorname{Lag}=\frac{1}{2}\|E\|^{2}-\Sigma_{\mathrm{i}} \alpha_{\mathrm{i}}\left(L_{\mathrm{i}}\left(E^{T} V_{\mathrm{i}}+B\right)-1\right) \alpha_{\mathrm{i}} \geq 0, \forall i \mathrm{~T}
$$

The optimization problem can be rewritten in terms of $\alpha_{i}$ as a result of set the imitative of the Lagrangian to ' 0 ':

$$
\begin{gathered}
\text { Maximize } \mathrm{E}(\alpha)=\sum_{\mathrm{i}} \alpha_{\mathrm{i}}-\frac{1}{2} \sum_{i, j} \alpha_{i} \alpha_{j} L_{i} L_{j} V_{i}^{T} V_{j} \\
\text { subject to } \alpha_{\mathrm{i}} \geq 0, \sum_{\mathrm{i}} \alpha_{\mathrm{i}} L_{\mathrm{i}}=0 \forall i
\end{gathered}
$$

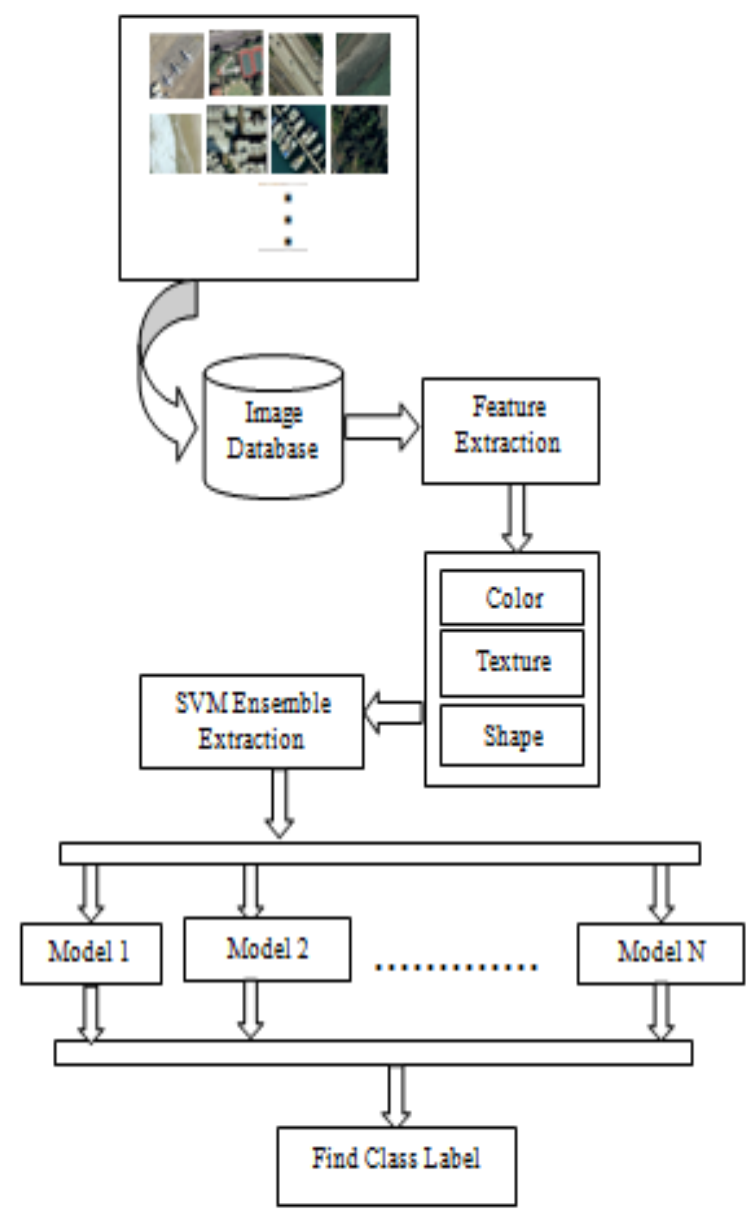

Fig. 3.Training Phase

\section{Testing Phase:}

The resulting classifier is applied to unlabelled images to decide whether the image belongs to the positive or the negative category. 
The label of L is simply obtained by computing as category 1 if the sum is positive, and category 2 otherwise.

$\mathrm{E}=1 . \quad \sum_{j=1}^{5} \alpha_{t_{j}}$ with 1. 1. $\quad(j=$ : indices of the $\mathrm{s}$ support vectors. Fig. 4 illustrates the testing phase of proposed model.

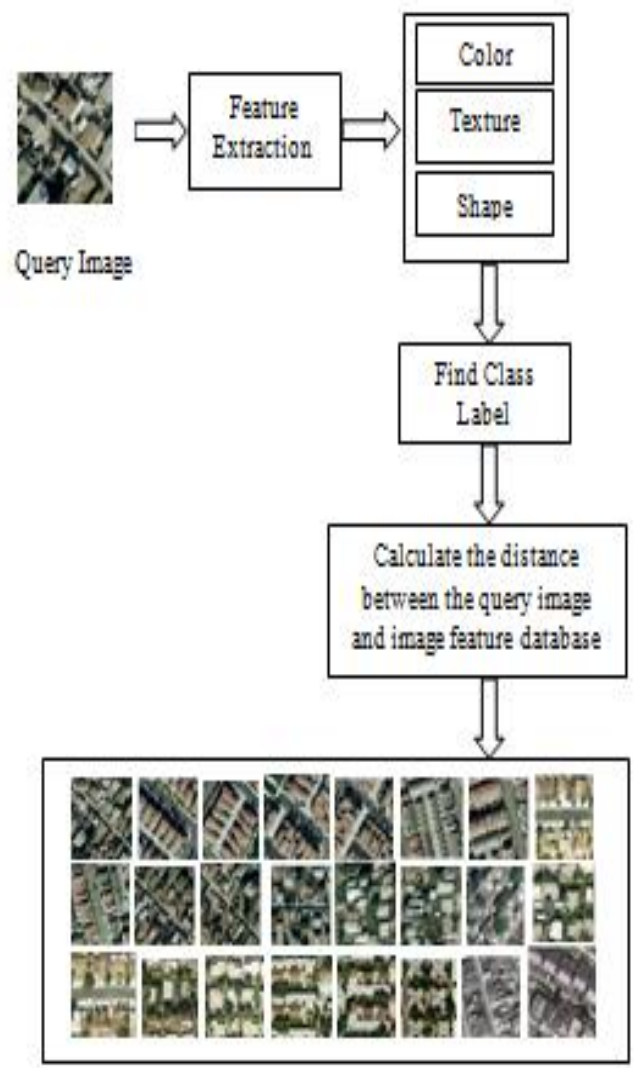

Relevant inages retrieved from the database

Fig. 4.Testing Phase

\section{EXPERIMENTAL RESULTS}

To estimate the proposed model, many experiments are developed and achievement is evaluated by differentiating the proposed model with existing models.

\section{A. Dataset Description}

This is a 21 class dataset of land images which is used for research purposes. Every group category contains 100 images of $256 \times 256$ pixels. Totally there are 2100 images in this dataset. The dataset images were gathering from the high resolution images collected from the regions around the country by USGS National Map Urban Area Imagery amassment.
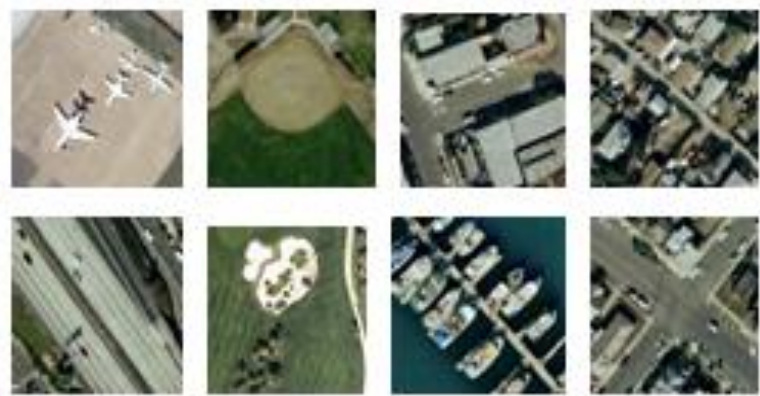

Fig. 5.Sample Images from UCMerced Dataset

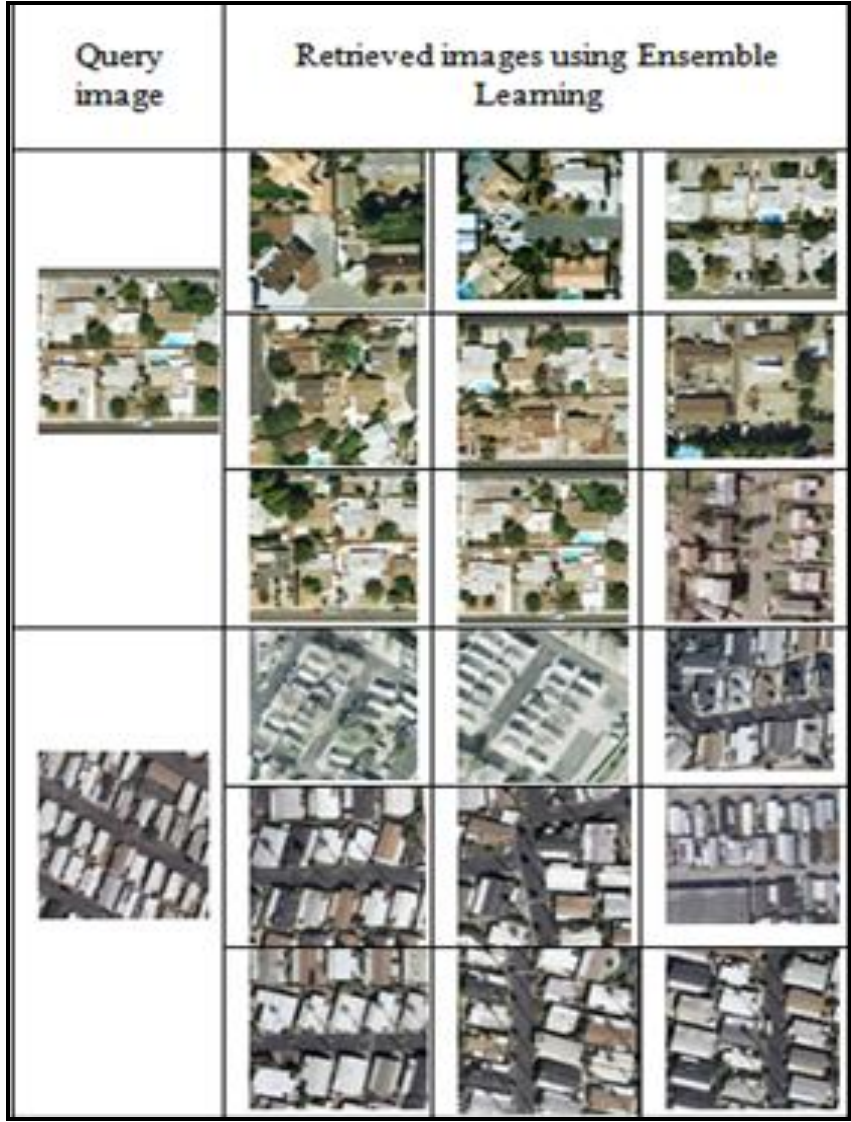

Fig. 6.Results of Sample Images fromUCMerced

The image retrieval dataset has been taken from UCMerced land use dataset that contains multiple satellite image categories of airplane images, agriculture images, baseball diamond images, beach images, buildings images, chaparral images, dense residential images, forest images, freeway images, golf course images, harbor images, intersection images, medium residential images, mobile home park images, overpass images, parking lot images, river images, runway images, sparse residential images, storage tanks images and tennis court images, whereas different numbers of images have been kept under the different image categories to form the final training image set. Images from the class categories airplane, baseball diamond, buildings, dense residential, freeway, golf course, harbor and intersection are shown in the Fig. 5. 
We choose the images randomly from each class categories of UCMerced dataset for training the SVM classifier and the rest for testing. From each class category, 80 images from dataset for training and 20 images from dataset for testing samples. Fig. 5 shows some sample images from the UCMerced land use dataset. The proposed ensemble learning approach has been found accurate with the accuracy level of more than $90 \%$ in comparison with the existing models.

\section{B. Performance Evaluation}

The efficiency of image retrieval systems are often preserved by using Precision and Recall measures. The formulations of these two measures are like the following definitions: Precision definition is equal to the ratio of the amount of relevant images retrieved to the amount of entire images retrieved from the database. The measurement Recall is described as the quantity of retrieved appropriate images over the sum of relevant images accessible in the image database.

\section{Precision-Recall}

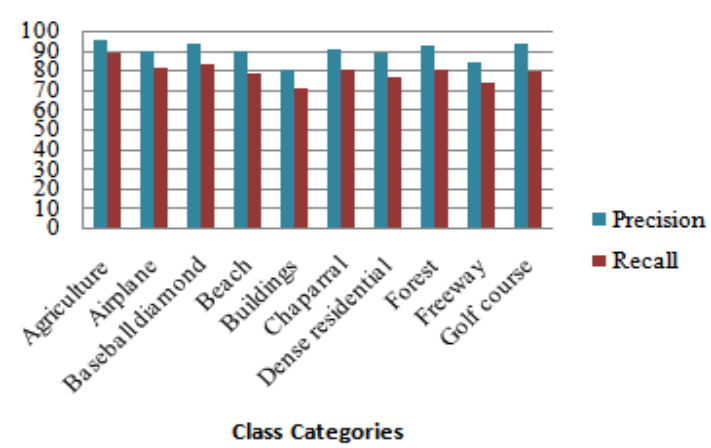

Fig. 7.Precision - Recall Values

Precision deals the truth or standard quality of the image retrieval process and Recall evaluates the completeness or amount of the images retrieval dealings. Every query images the two main measurements precision and recall values were predicted. The obtained high precision worth implies that additional relevant images are retrieved in our distinctive proposed ensemble learning method.

\section{Comparative Analysis}

The comparative analysis of SVM with some of the previous algorithms is shown below in graph format. In this proposed model the Support Vector Machine (SVM) is compared with different existing methods.

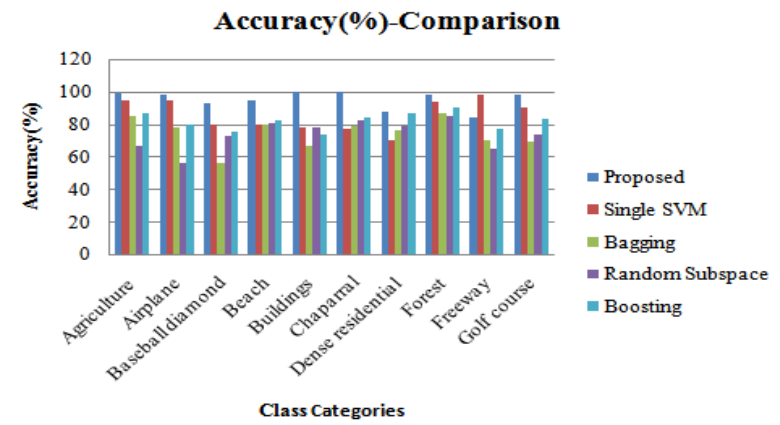

From the Fig. 8, SVM ensemble learning has the best average accuracy of $89.7 \%$ that shows SVM based ensemble model has a great performance results. So, SVM is absolutely a suggestive base learner. Boosting approach significantly boosts the results of random subspace in proposed SVM ensembel learning. The computational times for all existing methods are $40.5 \mathrm{sec}, 42.62 \mathrm{sec}$ and $58.51 \mathrm{sec}$ and SVM have $6.03 \mathrm{sec}$. This result is displayed in Fig.9.

\section{Time(in sec)- Comparison}

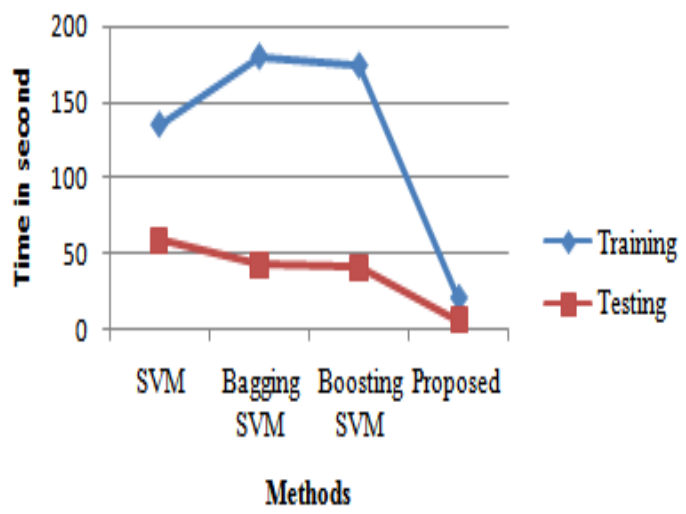

Fig. 9.Training and Testing Comparison

Compared with that of several other methods like Single SVM, Bagging and boosting, the time taken for training and testing is also excellent by using the proposed ensemble learning method.

\section{CONCLUSION}

Ensemble learning with SVM classifier is projected in this paper for content based image retrieval process. Partitioning the query image into data points using random subspace develop the performance results. Every sub-classifier is learned comparable image subspace. SVM sub-classifiers give excellent results and combined to generate an ensemble classifier.

Tentative outcome proves that our proposed approach preserve the information enclosed within image data and recover better retrieval results. At the same time as a basic classifier with the problem of curse of dimensionality is used, the proposed method can diminish the gap dimensionality and conquer the difficulty. Comparative analysis of various image retrieval algorithms has been done. From the comparative analysis it is observed that the retrieval time for a particular image have been reduced and the precision is increased. The primary aim of this paper is to represent the significance of ensemble learning with support vector machine in efficient retrieval of image.

Furthermore, it is achievable to get better image retrieval by using supplementary machine learning algorithms beside through decrease the retrieval time. Furthermore, it is possible to improve this retrieval performance by using other machine learning algorithms along with reduction in the retrieval time.

Fig. 8.Accuracy Comparison 


\section{REFERENCES}

1. R.C Gonzalez, R.E Woods, Digital Image Processing, 3rd Edition, 2010

2. Datta, Ritendra, et al. "Image Retrieval: Ideas, influences, and trends of the new age" ACM computing Surveys (CSUR)40.2 (2008)

3. Cortes, corinna and Vladmir Vapnik, "Support Vector networks", Machine learning, 20.3 (1995)

4. C. W. Deng, G. B. Huang, J. Xu, and J. X. Tang, "Extreme learning machines: new trends and applications," Science China Information Sciences, vol. 58, no. 2, pp. 1-16, 2015.

5. L. Breiman, "Bagging predictors", Machine learning, vol. 24, no. 2, (1996), pp. 123-140.

6. Dongping Tian, "Support Vector Machine for Content-based Image Retrieval: A Comprehensive Overview", Journal of Information Hiding and Multimedia Signal Processing, Volume 9, Number 6, November 2018.

7. Baohua Yuan; Shijin Li; Ning Li, "Multiscale deep features learning for land-use scene recognition", Journal of Applied Remote Sensing, 12(1), 015010 (2018). https://doi.org/10.1117/1.JRS.12.015010

8. X. Li, L. Wang, \& E. Sung, "AdaBoost with SVM-based component classifiers", Engineering Applications of Artificial Intelligence, vol. 21, no. 5, (2008), pp. 785-795.

9. Aun Irtaza1, Syed M. Adnan, Khawaja Tehseen Ahmed, Arfan Jaffar, Ahmad Khan, Ali Javed and Muhammad Tariq Mahmood, "An Ensemble Based Evolutionary Approach to the Class Imbalance Problem with Applications in CBIR", Applied Sciences. 2018, 8, 495; doi:10.3390/app8040495.

10. Haifeng Wang, Bichen Zheng, Sang Won Yoon, Hoo Sang Ko, "A support vector machine-based ensemble algorithm for breast cancer diagnosis", European Journal of Operational Research 267 (2018) 687-699.

11. Jianrong Yao, Cheng Lian, "A New Ensemble Model based Support Vector Machine for Credit Assessing", International Journal of Grid and Distributed Computing Vol. 9, No. 6 (2016), pp.159-168.

12. Min-Wei Huang, Chih-Wen Chen, Wei-Chao Lin, Shih-Wen Ke, Chih-Fong Tsai, "SVM and SVM Ensembles in Breast Cancer Prediction", PLOS ONE | DOI:10.1371/journal.pone.0161501 January 6, 2017.

13. Ela Yildizer, Ali Metin Balci, Mohammad Hassan, Reda Alhajj, "Efficient content-based image retrieval using Multiple Support Vector Machines Ensemble", Expert Systems with Applications 39 (2012) 2385-2396.

14. Garima Gupta, and Manish Dixit, "CBIR on Biometric Application using Hough Transform with DCD, DWT Features and SVM Classification", International Journal of Engineering and Innovative Technology, ISSN: 2277-3754, Volume 4, Issue 12, June 2016.

15. Pradnya Vikhar, and Pravin Karde, "Improved CBIR System using Edge Histogram Descriptor (EHD) and Support Vector Machine (SVM)", International Conference on ICT in Business Industry \& Government (ICTBIG), 978-1-5090-5515-9/16/\$31.00 @2016 IEEE.

16. Katta Sugamya, Suresh Pabboju, and Dr. A. Vinaya Babu, "A CBIR Classification using Support Vector Machines", 978-1-4673-8810-8/16/\$31.00 @2016 IEEE.

17. Navneet Kaur, Sonika Jindal, and Bhavneet Kaur, "Relevance Feedback Based CBIR System Using SVM and Bayes Classifier", Second International Conference on Computational Intelligence \& Communication Technology, 978-1-5090-0210-8/16 \$31.00 @ 2016 IEEE.

18. G., Wang, \& J. Ma, "A hybrid ensemble approach for enterprise credit risk assessment based on Support Vector Machine", Expert Systems with Applications, vol. 39, no. 5, (2012), pp. 5325-5331.

19. Yang, Y. and Newsam, S. "Bag-of-visual-words and spatial extensions for land-use classification", Proceedings of the 18th SIGSPATIAL international conference on advances in geographic information systems, 2010, 270-279.

20. Pouria Sadeghi-Tehran, Plamen Angelov, Nicolas Virlet and Malcolm J. Hawkesford, "Scalable Database Indexing and Fast Image Retrieval Based on Deep Learning and Hierarchically Nested Structure Applied to Remote Sensing and Plant Biology", Journal of Imaging, 2019, 5, 33. doi:10.3390/jimaging5030033.

21. Lu Xu, Dongping Ming, Wen Zhou, Hanqing Bao, Yangyang Chen and Xiao Ling, "Farmland Extraction from High Spatial Resolution Remote Sensing Images Based on Stratified Scale Pre-Estimation", Remote Sens. 2019, 11, 108; doi:10.3390/rs11020108

\section{AUTHORS PROFILE}

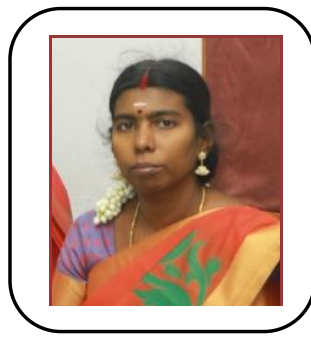

P.K.KAVITHA, has received her BSc. Mathematics degree in 1998, MCA degree in 2001 from SFR College, Sivakasi, affiliated to Madurai Kamaraj University, Madurai. In 2016, she received the M.Phil degree in computer science from VPMM Arts and Science College for Women, Krishnankoil, affiliated to Madurai Kamaraj University, Madurai. Currently, she is a full time Research scholar with University Research Fellowship in the Department of Computer Applications at Kalasalingam Academy of Research and Education, Krishnankoil, Tamil Nadu, India. Her research interests include Image Processing, Machine Learning and Cryptography.

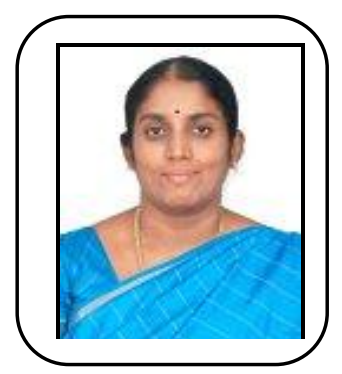

P. VIDHYA SARASWATHI has received her MCA degree in 1999, M.Phil (Computer Science) degree in 2007 from Madurai Kamaraj University and Ph. D from Kalasalingam Academy of Research and Education. She worked as a Lecturer at A.K.D. Dharmaraja Womens College, Rajapalayam, Tamilnadu, India, between 2001 and 2008. Currently, She is working as a Assistant Professor and Head of the Department of Computer Science and Information Technology at Kalasalingam Academy of Research and Education, Krishnankoil, Tamil Nadu, India. Her areas of interests include Cryptography, Image Processing, Machine Learning, Bioinformatics and Cloud computing. 